\title{
SEMG activity of jaw-closing muscles during biting with different unilateral occlusal supports
}

M.-Q. WANG*, J.-J. HE*, J.-H. ZHANG*, K. WANG ${ }^{\dagger}$, P. SVENSSON\& \& S. E. WID MALM II *Department of Oral Anatomy and Physiology and TMD, School of Stomatology, Fourth Military Medical University, Xi'an, Shanxi Province, China, ${ }^{\dagger}$ Center for Sensory-Motor Interaction, Aalborg University, Fredrik Bajers Vej, DK, Aalborg Øst, ${ }^{\ddagger}$ Department of Oral $\theta$ Maxillofacial Surgery, Aalborg Hospital, ${ }^{\S}$ Department of Clinical Oral Physiology, School of Dentistry, University of Aarhus, Denmark and "Department of Biological and Materials Sciences, Division of Prosthodontics, University of Michigan, Ann Arbor, MI, USA; and Visiting Professor, Departments of Oral Anatomy and Physiology and TMD, School of Stomatology, Fourth Military Medical University, Xi'an, Shanxi Province, China

SUMMARY The aim of this study was to test the hypothesis that experimental and reversible changes of occlusion affect the levels of surface electromyographic (SEMG) activity in the anterior temporalis and masseter areas during unilateral maximal voluntary biting (MVB) in centric and eccentric position. Changes were achieved by letting 21 healthy subjects bite with and without a cotton roll between the teeth. The placement alternated between sides and between premolar and molar areas. The SEMG activity level was lower when biting in eccentric position without than with a cotton roll between teeth $(P<0.043)$. It was always lower with premolar than with molar support when biting with a cotton roll $(P<0.013)$. In the anterior temporalis areas, the SEMG activity was always lower on the balancing than on the working side $(P<0.001)$. Such a difference was also found in the masseter areas but only during molar-supported centric biting $(P=0.024)$. No differences were found when comparing the SEMG levels in masseter areas between centric and eccentric biting $(P>0.05)$. In the anterior temporalis area, the balancing side SEMG activity was lower in eccentric than in centric but only in molar-supported biting $(P=0.026)$. These results support that the masseter and anterior temporalis muscles have different roles in keeping the mandible in balance during unilateral supported MVB. Changes in occlusal stability achieved by biting with versus without a cotton roll were found to affect the SEMG activity levels.

KEYWORDS: electromyography, dental-occlusion, jawclosing muscles, mandibular position, trigeminal neurophysiology

Accepted for publication 11 April 2010

\section{Introduction}

The degree of the maximal voluntary biting (MVB), and how it is affected by centric versus eccentric mandibular position, by the location of a bolus and by occlusal stability, can be approximated by measuring the degree of electromyographic (EMG) activity as recorded with surface electrodes (1-11). Recently, cotton rolls were used to study the effect of experimentally changed location of occlusal load on surface electromyographic (SEMG) activity during centric maximal voluntary clenching. It was found that with cotton rolls placed bilaterally in the premolar instead of the molar areas, the SEMG activity decreased both in the masseter and in the anterior temporalis areas. When biting with the mandible in centric position with a cotton roll on one side, the SEMG activity decreased both in working and balancing masseter areas. In the temporalis area, however, the SEMG activity decreased only on the balancing side (8). This indicates strongly that changes in the placement of a bolus have different effects on the degree and symmetry of masseter and temporalis 
muscle activity during centric maximal voluntary clenching. However, during chewing, the bolus is mostly placed on the left or the right side and the mandible is often positioned more or less eccentric. Keeping the mandible in a balanced position during unilateral clenching on a tough food bolus in eccentric position is a task that can be expected to need well-coordinated muscle activity. Therefore, further research is motivated to test if the elevator muscles are affected in the same way during MVB in eccentric as in centric positions.

The term occlusion which, according to common definitions (12), refers to 'The act of closure or state of being closed' will in this article be used as referring to conditions when the subject had contacts between opposing teeth with or without a cotton roll being inserted. Generally, the studies indicate that the SEMG levels are increased when subjects bite on a soft surface compared to a hard surface, such as experimental interferences (10), plastic appliances (11, 13-17), or on force transducers (18-22), probably because of a better stability and more contact points being obtained (8).

The jaw muscle SEMG activity degree at clenching is affected by factors such as the mandibular position (17, $23,24)$, the number and location of the occlusal contacts $(10,11)$, occlusal support distribution (10, $25,26)$, the direction of force vectors (27), the level of biting force (2), contact versus no contact between premolars in centric versus eccentric position (28) and age (29). Both human and animal studies have demonstrated distinct differences between women and men in terms of jaw movements (30), muscle fibre composition, twitch forces and torques (31). Several other factors such as pain and muscle fatigue $(32,33)$ may also exert a control on the jaw-closing muscle.

Most of the clenching activity recorded with surface electrodes in the temporalis area may be from the temporalis muscle and most in the masseter area may be from the masseter muscle. It is, however, realized that the individual SEMG recordings mostly contain a mixture of activity from several jaw muscles and may be contaminated with facial muscle activity. Therefore, the label 'muscle area' is preferred here. If the localization to specific individual muscles is desirable, the recordings have to be complemented with intra-muscular electrodes $(34,35)$.

Keeping the mandible in a balanced situation when chewing tough food unilaterally can be expected to need well-coordinated elevator muscle activity. The aim of this study was to test for differences in SEMG activity in the masseter and the anterior temporalis areas during MVB with unilateral occlusal support with and without modification by a cotton roll, and for the changes of the mandibular position from centric to eccentric and the changes of the location of the occlusal support from molar to premolar. The response to changes from biting with, to biting without, an inserted cotton roll represents here changes from a stable to an unstable occlusion relation. The hypothesis was that the jaw-closing muscle activity degree, as recorded by SEMG in the masseter and the anterior temporalis areas, was affected by changes in occlusal stability, changes in mandibular horizontal positions and changes in the occlusal support location.

\section{Materials and methods}

Twenty-one healthy students, 11 men and 10 women, aged 22-24, mean $21 \pm 0 \cdot 5$ (SD), participated in this study. Before testing, the subjects were independently examined by two trained clinicians. The subjects had at least 28 permanent teeth with group function relation during right and left lateral movements. None had occlusal contacts on the non-working side while biting in eccentric position. All chewed alternating between sides. No one had any signs of temporomandibular disorders (36). All volunteers gave informed consent, and the study was approved by the local ethical committee of the Fourth Military Medical University.

The SEMG activity was recorded in accordance with our previous experiment (8). Briefly, a commercially available EMG equipment (EM-2*) was used with surface electrodes* placed in the centre of the masseter and anterior temporalis areas parallel to the direction of the main superficial muscle fibre bundles. The interelectrode distance was $20 \mathrm{~mm}$ in each pair, and a common reference electrode was placed in the back of neck as reported in a previous study. The high- and lowpass SEMG filters were set at 10 and $1000 \mathrm{~Hz}$. The root mean square (RMS) values of the SEMG activity recordings were calculated. The subjects were allowed to practice prior to the start of the experiment. They were instructed to keep looking at a mark placed $2 \mathrm{~m}$ away at eye level, keeping the head in a steady upright position, and were encouraged verbally to clench with maximal effort in the various tests. Each SEMG recording of MVB lasted $5 \mathrm{~s}$ with 1- to 2-min intervals between

*EM-2, Myotronic Co., Seattle, WA, USA. 


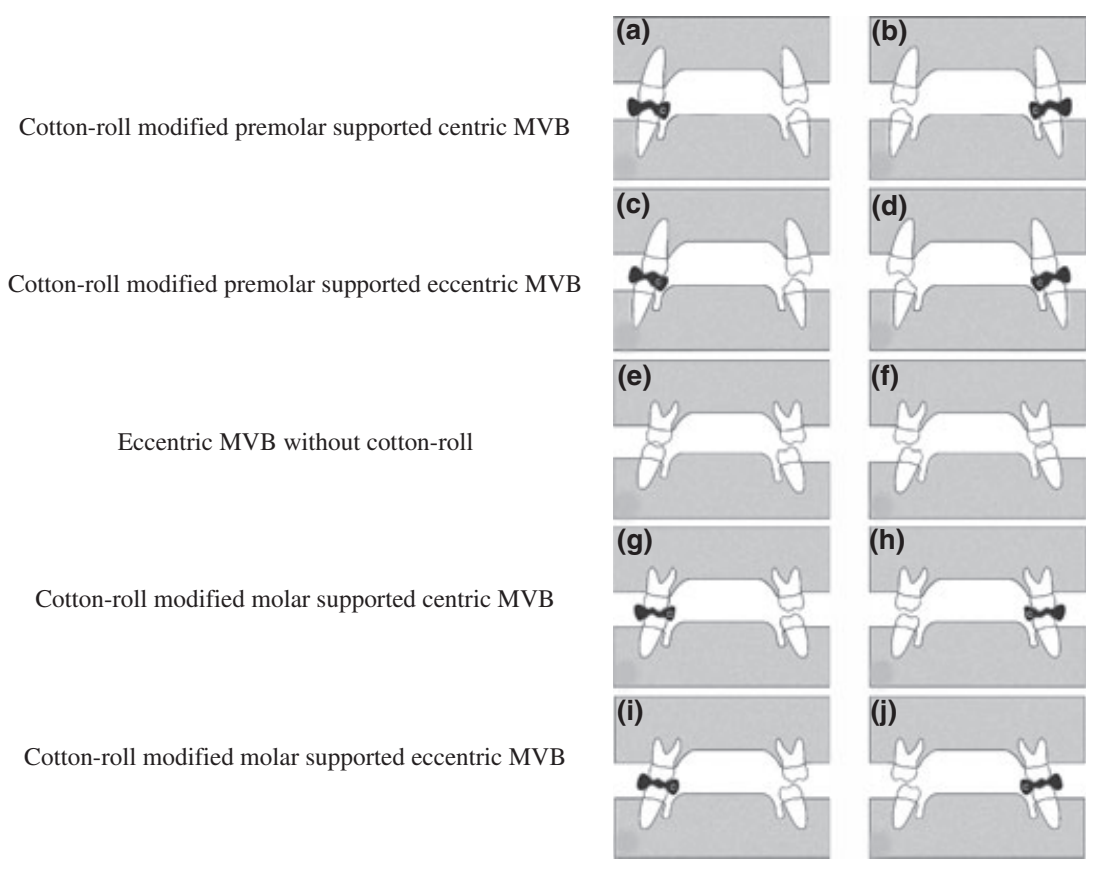

Fig. 1. Diagram of the five occlusion conditions in present unilateral biting tasks. The cotton rolls are illustrated as black dumb-bells. They were put either in the premolar (a, b, c, d) or the molar areas ( $g, h, i, j)$, either on the right side (a, c, g, i) or on the left side (b, d, h, j). The mandible was either in a centric $(\mathrm{a}-\mathrm{b}, \mathrm{g}-\mathrm{h})$ or an eccentric position $(\mathrm{c}-\mathrm{d}, \mathrm{e}-\mathrm{f}, \mathrm{i}-\mathrm{j})$. The eccentric positions without cotton roll are illustrated in e-f. MVB, maximal voluntary biting.

repetitions to avoid muscle fatigue. The interval between two different tasks was 5 min and the total recording time for each student was about $30 \mathrm{~min}$. The mean RMS SEMG values of two repeated recordings were used in the statistical analysis.

As described in a previous study, cotton rolls $(17 \mathrm{~mm}$ in length and $10 \mathrm{~mm}$ diameter) were used as modifiers intended to provide a more stable occlusion relation by preventing potentially disturbing contacts between opposing teeth and increasing the contact area (8). In this view, biting without a cotton roll in eccentric position is unstable. The bite opening was estimated with a ruler to be approximately $1 \mathrm{~mm}$ when biting on the cotton roll and all subjects confirmed that there were no contacts on the balancing side.

The long axes of the cotton roll(s) were in the buccallingual direction. The cotton rolls were positioned on the mandibular dentition either on the right or on the left side, and either in the molar (molar-supported clenching) or premolar (premolar-supported clenching) region. The mandible was positioned either at a centric or at an eccentric position (Fig. 1). The SEMG activity during MVB was also recorded with the mandible in the same eccentric position and with the teeth occluding but without a cotton roll inserted. Thus, as illustrated in
Fig. 1, MVB was recorded in a total of 10 (five pairs of right and left) different occlusal positions in randomized order when the mandible was either in a centric or an eccentric position with a cotton roll placed on one side, either in the molar or the premolar area. Recording was also made with the mandible in the eccentric position but without a cotton roll.

The SEMG recordings were normalized within each subject by calculating the ratios between each test recording and a reference, here chosen as a recording from clenching with MVB in centric intercuspal position without cotton rolls.

The statistical analysis was performed using the SPSS 11.0 package $^{\dagger}$. Student $t$-test was used to compare activity levels during different biting tasks (as indicated in Fig. 1) between genders. No significant differences were found. Therefore, data from male and female subjects were pooled before further analysis. Paired Student $t$-test was used to compare the SEMG levels between contractions when the cotton roll was placed either on the right or the left side. No significant differences were found. Therefore, the working side data were pooled and so were the balancing side data

${ }^{\dagger}$ SPSS Inc., Chicago, IL, USA. 
Table 1. Root mean square values (mean $\pm \mathrm{SD}$ ) of the SEMG activity in areas of anterior temporalis (TA) and masseter (MM) in $\mu \mathrm{V}$ during maximal voluntary biting (MVB) in centric and eccentric positions with cotton rolls being inserted between upper and lower dentitions and without cotton rolls. $(n=21)$

\begin{tabular}{|c|c|c|c|c|c|c|c|}
\hline & & \multicolumn{2}{|l|}{ TA } & \multirow[b]{2}{*}{$P$ value } & \multicolumn{2}{|l|}{ MM } & \multirow[b]{2}{*}{$P$ value } \\
\hline & & $\begin{array}{l}\text { Working } \\
\text { side }\end{array}$ & $\begin{array}{l}\text { Balancing } \\
\text { side }\end{array}$ & & $\begin{array}{l}\text { Working } \\
\text { side }\end{array}$ & $\begin{array}{l}\text { Balancing } \\
\text { side }\end{array}$ & \\
\hline \multirow{3}{*}{$\begin{array}{l}\text { Mandible in centric with } \\
\text { cotton roll loading on }\end{array}$} & Premolar & $143 \pm 53 \cdot 3$ & $62 \pm 53 \cdot 2$ & $<0 \cdot 000$ & $137 \pm 63 \cdot 3$ & $149 \pm 52 \cdot 1$ & $0 \cdot 119$ \\
\hline & Molar & $194 \pm 58 \cdot 2$ & $153 \pm 58 \cdot 9$ & $<0 \cdot 000$ & $209 \pm 51 \cdot 5$ & $182 \pm 55 \cdot 7$ & $<0 \cdot 000$ \\
\hline & Premolar & $155 \pm 59 \cdot 9$ & $76 \pm 51 \cdot 3$ & $<0 \cdot 000$ & $142 \pm 62 \cdot 8$ & $151 \pm 60 \cdot 7$ & $0 \cdot 244$ \\
\hline $\begin{array}{l}\text { Mandible in eccentric with } \\
\text { cotton roll loading on }\end{array}$ & Molar & $190 \pm 51 \cdot 8$ & $127 \pm 63$ & $<0.000$ & $198 \pm 44 \cdot 8$ & $174 \pm 46 \cdot 9$ & $0 \cdot 002$ \\
\hline Mandible without cotton-roll & Eccentric & $\begin{array}{l}132 \pm 71 \cdot 2 \\
\text { LTA }\end{array}$ & $\begin{array}{l}34 \pm 25 \cdot 6 \\
\text { RTA }\end{array}$ & $<0.000$ & $\begin{array}{l}76 \pm 53 \cdot 3 \\
\mathrm{LMM}\end{array}$ & $\begin{array}{l}107 \pm 59 \cdot 9 \\
\mathrm{RMM}\end{array}$ & $0 \cdot 001$ \\
\hline Centric biting without cotton roll & & $205 \pm 65 \cdot 7$ & $203 \pm 60 \cdot 5$ & $0 \cdot 798$ & $235 \pm 62 \cdot 4$ & $235 \pm 48 \cdot 8$ & $0 \cdot 993$ \\
\hline
\end{tabular}

for each muscle area before further comparisons. A $t$ test for dependent measures was used to compare the ratios between muscle activity levels with versus without a cotton roll in eccentric position, with a cotton roll in centric versus eccentric positions, with a cotton roll in the molar versus the premolar area. Comparisons were also made between working and balancing side activity levels. $P$-values were considered significant when $<0 \cdot 05$. listed in Table 1 and those based on normalized SEMG recordings are shown in Fig. 2.

\section{With versus without cotton rolls in eccentric position}

The MVB activity was lower in eccentric position without a cotton roll in all four muscle areas than in MVB in eccentric position with cotton rolls at premolar or molar area $(P<0.005)$.

\section{Results}

Centric versus eccentric with the insertion of a cotton rolls

The results of biting with maximal voluntary activity (MVB) based on original 'raw' SEMG recordings are

Balancing side activity in the temporalis area was lower in eccentric than in centric in molar-supported MVB

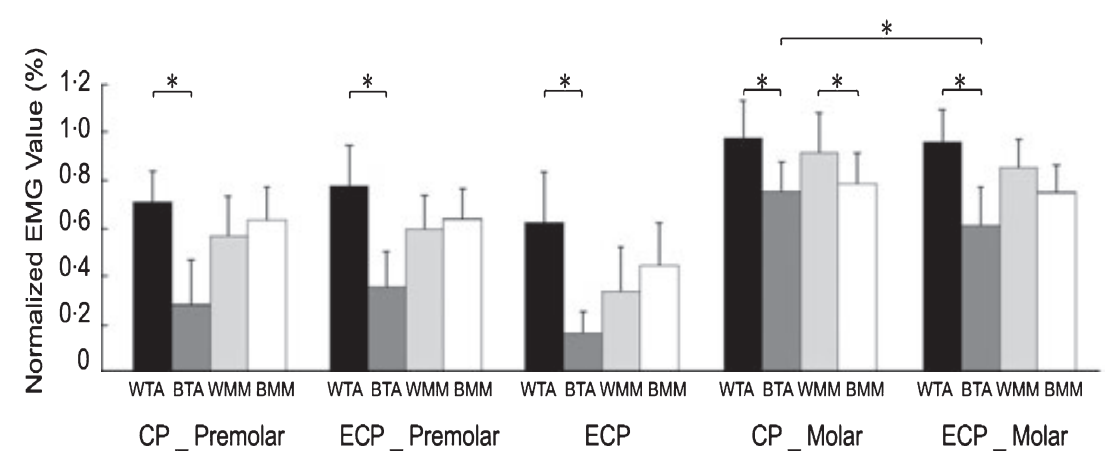

Fig. 2. The means of the normalized SEMG values $(n=21$; mean $+\mathrm{SD})$ from masseter and anterior temporalis areas during unilateral biting. The four columns in each of the five groups represent the normalized data from maximal voluntary biting (MVB) with cotton rolls placed at the first premolar or molar region with the mandible in centric or eccentric position, and during unilateral biting in eccentric position without cotton roll(s). WTA, working side anterior temporalis area; WMM, working side masseter area; BTA, balancing side anterior temporalis area; $\mathrm{BMM}$, balancing side masseter area; $\mathrm{CP}_{-}$Premolar, MVB in centric position with a cotton roll placed at the first premolar area; ECP_Premolar, MVB in eccentric position with a cotton roll placed at the first premolar area; ECP, MVB in eccentric position without cotton rolls between upper and lower teeth; $C_{-}$_Molar, MVB in centric position with a cotton roll placed at the first molar area; ECP_Molar, MVB in eccentric position with a cotton roll placed at the first molar area. ${ }^{*} P<0 \cdot 05$. Comparing to centric and eccentric biting with cotton rolls all the values of the four muscles during biting in eccentric position without cotton rolls were lower. In addition, compared to centric molarsupported biting, all the values of the four muscles during premolar-supported MVB were lower. 
$(P=0 \cdot 003)$. No other differences were found in this test.

\section{Premolar-versus molar-supported biting}

The activity was lower in premolar- than in molarsupported biting in masseter and anterior temporalis areas during centric and eccentric MVB $(P<0 \cdot 003)$.

\section{Working side versus balancing side}

The activity was lower on the balancing than on the working side in the anterior temporalis area $(P<0.001)$ in all of the five tested occlusal conditions. In the masseter areas, this difference was found only during centric molar-supported MVB $(P=0 \cdot 006)$.

\section{Discussion}

The results imply that a powerful elevator activity was supported by a stable occlusal relation because when improved stability of occlusal relations was provided by the cotton rolls, the SEMG activity degree was as high in the masseter and the anterior temporalis areas at eccentric position as at centric position. When biting in the same eccentric position with an unstable relation, i.e., without insertion of a cotton roll, the SEMG activity degrees were at their lowest levels in all four areas. No gender differences were found. These results are in agreement with Jimenez (6) who found that occlusal stability has more influence than mandibular sagittal position on masseter and anterior temporalis MVB SEMG levels. Supportively, MVB SEMG activity in masseter areas of healthy subjects with natural dentitions has been reported to be lower when biting in edge-to-edge lateral contact position than when biting in the intercuspal position (24), but when the cotton rolls were inserted, as in this study, no such effect was observed in the masseter muscle area.

Baba et al. (23) reported that clenching with only bilateral first premolar support caused a larger upward movement of the mandible in the posterior region than biting with bilateral second molar support. They also reported that clenching on unilateral occlusal stops caused an upward movement on the contralateral side of the mandible. These results showed that when the clenching load is moved from molar to premolar regions, the SEMG activity decreases in all areas, and when clenched unilaterally, the working side SEMG activity in masseter area is as low as the balancing side. These results suggest a protective reaction of the masseter muscle by lowering its activity in avoiding harmful displacement of the mandible during clenching in an unstable condition (37), and are in accordance with earlier reports about MVB in healthy subjects in closed position $(8,24)$ and in unilateral biting on a bite-force transducer (38). The anterior temporalis areas activity, however, differed from that in the masseter areas. The SEMG activity levels were always lower on the balancing side than on the working side in the anterior temporalis. In MVB with molar support, the SEMG activity in balancing side anterior temporalis area was lower when biting in eccentric than in centric position, while no such differences were found in the masseter areas.

The individual role of the many subdivisions of the masseter and temporalis muscles in keeping the mandible in balance during biting and chewing cannot be analysed by SEMG only. Needle or wire electrodes for EMG recordings are, however, not realistic methods in studies on large groups of human subjects because of the pain and possible trauma such electrodes may cause. Surface EMG does still give valuable information as long as it is kept in mind that the recordings represent composite activity patterns from groups of muscles where the degree of individual muscles, or subdivisions of muscles, cannot be accurately defined. With this in mind, it is still reasonable to conclude that the differences between recorded SEMG patterns in this study strongly indicate that the roles of the temporalis and masseter muscles in keeping the mandibular balance during unilateral MVB are different. It should also be noted that it is a matter of discussion if normalization of EMG data should be performed but several other investigations have recommended this approach (for a discussion, see ref 39). It is reasonable to conclude that the anterior temporalis and masseter muscles have functional differences during chewing, which is in agreement with van der Bilt et al. (22).

Future studies on subjects who prefer one and the same side for unilateral chewing versus those who alternate between sides are motivated to study if asymmetrical jaw elevator activity has negative longterm effects on jaw muscle and temporomandibular joint function. A simultaneous measuring of the mandibular movement during biting like that reported by Baba et al. (23) would provide more information. Finally, it should be recognized that placement of the cotton roll between the teeth could have changed the 
biomechanics of the temporomandibular joint with a different afferent input to the motorneuron pool and that this might have influenced the activation of the elevator muscles. However, because of small change in the angle of the condyle in the fossa that the cotton roll may have caused this influence is not likely to have played a major role. It might have been desirable to simultaneously record the biting force with a transducer to monitor the biting force levels. A 'naked' bite transducer may not provide as stable and comfortable contact relations as a cotton roll and further studies are planned where a bite transducers wrapped in soft closing will be used.

\section{Acknowledgment}

This study was supported by the National Nature Science Foundation of China (NSFC) No. 30540130469. The participation of volunteers is greatly appreciated.

\section{References}

1. Nickel JC, Iwasaki LR, Walker RD, McLachlan KR, McCall WD Jr. Human masticatory muscle forces during static biting. J Dent Res. 2003;82:212-217.

2. Naeije M, McCarroll RS, Weijs WA. Electromyographic activity of the human masticatory muscles during submaximal clenching in the inter-cuspal position. J Oral Rehabil. 1989;16:63-70.

3. Bakke M, Michler L, Møller E. Occlusal control of mandibular elevator muscles. Scand J Dent Res. 1992;100:284-291.

4. Bakke M. Mandibular elevator muscles: physiology, action, and effect of dental occlusion. Scand J Dent Res. 1993;101:314-331.

5. Iwasaki LR, Petsche PE, McCall WD Jr, Marx D, Nickel JC. Neuromuscular objectives of the human masticatory apparatus during static biting. Arch Oral Biol. 2003;48:767-777.

6. Jimenez ID. Dental stability and maximal masticatory muscle activity. J Oral Rehabil. 1987;14:591-598.

7. Jimenez ID. Electromyography of masticatory muscles in three jaw registration positions. Am J Orthod Dentofacial Orthop. 1989;95:282-288.

8. Wang MQ, He JJ, Wang K, Svensson P. Influence of changing occlusal support on jaw-closing muscle electromyographic activity in healthy men and women. Acta Odont Scand. 2009;67:187-192.

9. Holmgren K, Sheikholeslam A. Occlusal adjustment and myoelectric activity of the jaw elevator muscles in patients with nocturnal bruxism and craniomandibular disorders. Scand J Dent Res. 1994;102:238-243.

10. MacDonald JW, Hannam AG. Relationship between occlusal contacts and jaw-closing muscle activity during tooth clenching: Part I. J Prosthet Dent. 1984;52:718-728.
11. Manns A, Miralles R, Valdivia J, Bull R. Influence of variation in antero-posterior occlusal contacts on electromyographic activity. J Prosthet Dent. 1989;61:617-623.

12. Zwemer TJ. Boucher's clinical dental terminology. 4th edn, Mosby, St. Louis; 1993, p210.

13. Roark AL, Glaros AG, O'Mahony AM. Effects of interocclusal appliances on EMG activity during parafunctional tooth contact. J Oral Rehabil. 2003;30:573-577.

14. Fitins D, Sheikholeslam A. Effect of canine guidance of maxillary occlusal splint on level of activation of masticatory muscles. Swed Dent J. 1993;17:235-241.

15. Wood WW, Tobias DL. EMG response to alteration of tooth contacts on occlusal splints during maximal clenching. J Prosthet Dent. 1984;51:394-396.

16. Castroflorio T, Talpone F, Deregibus A, Piancino MG, Bracco P. Effects of a functional appliance on masticatory muscles of young adults suffering from muscle-related temporomandibular disorders. J Oral Rehabil. 2004;31:524-529.

17. Ceneviz C, Mehta NR, Forgione A, Sands MJ, Abdallah EF, Lobo Lobo $\mathrm{S}$ et al. The immediate effect of changing mandibular position on the EMG activity of the masseter, temporalis, sternocleidomastoid, and trapezius muscles. Cranio. 2006;24:237-244.

18. Mao J, Osborn JW. Direction of a bite force determines the pattern of activity in jaw-closing muscles. J Dent Res. 1994;73:1112-1120.

19. van Eijden TM. Jaw muscle activity in relation to the direction and point of application of bite force. $J$ Dent Res. 1990;69:901-905.

20. Chandu A, Suvinen TI, Reade PC, Borromeo GL. The effect of an interocclusal appliance on bite force and masseter electromyography in asymptomatic subjects and patients with temporomandibular pain and dysfunction. J Oral Rehabil. 2004;31:530-537.

21. Slagter AP, Bosman F, van der Glas HW, van der Bilt A. Human jaw-elevator muscle activity and food comminution in the dentate and edentulous state. Arch Oral Biol. 1993;38:195-205.

22. van der Bilt A, Tekamp A, van der Glas H, Abbink J. Bite force and electromyograpy during maximum unilateral and bilateral clenching. Eur J Oral Sci. 2008;116:217-222.

23. Baba K, Akishige S, Yaka T, Ai M. Influence of alteration of occlusal relationship on activity of jaw closing muscles and mandibular movement during submaximal clenching. J Oral Rehabil. 2000;27:793-801.

24. Campillo MJ, Miralles R, Santander H, Valenzuela S, Fresno MJ, Fuentes A et al. Influence of laterotrusive occlusal scheme on bilateral masseter EMG activity during clenching and grinding. Cranio. 2008;26:263-273.

25. Hekneby M. The load of the temporomandibular joint: physical calculations and analyses. J Prosthet Dent. 1974;31:303-312.

26. Baba K, Yugami K, Akishige S, Ai M. Immediate effect of occlusal contact pattern in lateral jaw position on the EMG activity in jaw-elevator muscles in humans. Int J Prosthodont. 2000; 13:500-505.

27. van Eijden TM. Three-dimensional analyses of human biteforce magnitude and moment. Arch Oral Biol. 1991;36: $535-539$. 
28. Widmalm SE, Ericsson SG. Maximal bite force with centric and eccentric load. J Oral Rehabil. 1982;9:445-450.

29. Peyron MA, Blanc O, Lund JP, Woda A. Influence of age on adaptability of human mastication. J Neurophysiol. 2004; 92:773-779.

30. Gerstner GE, Parekh VV. Evidence of sex-specific differences in masticatory jaw movement patterns. J Dent Res. 1997; 76:796-806.

31. English AW, Widmer CG. Sex differences in rabbit masseter muscle function. Cells Tissues Organs. 2003;174:87-96.

32. Karibe H, Goddard G, Gear RW. Sex differences in masticatory muscle pain after chewing. J Dent Res. 2003;82:112-116.

33. Christensen LV. Experimental teeth clenching in man. Swed Dent J Suppl. 1989;60:1-66.

34. Schumacher GH. Funktionelle Morphologie der Kaumuskulatur. pub VEB Gustav Fischer Verlag, Jena; 1961, pp 1-53.

35. Basmajian JV. Electromyography - Dynamic gross anatomy: a review. Am J Anat. 1980;159:245-260.
36. National Institutes of Health Technology Assessment Conference Statement. Management of temporomandibular disorders. J Am Dent Assoc. 1996;127:1595-1599.

37. Pröschel PA, Raum J. Preconditions for estimation of masticatory forces from dynamic EMG and isometric bite forceactivity relations of elevator muscles. Int $\mathrm{J}$ Prosthodont. 2001;14:563-569.

38. Kozawa T, Igarashi Y, Yamashita S. Posterior occlusal support and bite force influence on the mandibular position. Eur J Prosthodont Restor Dent. 2003;11:33-40.

39. Ferrario VF, Sforza C, Tartaglia GM. Commentary to Suvinen and Kempaininen. J Oral Rehabil. 2009;36:9-10.

Correspondence: Mei-Qing Wang, Professor and Chair, Dept. of Oral Anatomy and Physiology and TMD, School of Stomatology, Fourth Military Medical University, Xi'an, Shanxi Province, China.

E-mail: mqwang@fmmu.edu.cn 